Original Research Article

\title{
Antimicrobial activity of Allium cepa and Cinnamomum zeylanicum against common bacteria causing urinary tract infections: in vitro study
}

\author{
Mayank R. Dhore*, Asha R. Jha
}

Department of Pharmacology, JNMC, DMIMS, Sawangi Meghe, Wardha, Maharashtra, India

Received: 01 April 2019 Accepted: 07 May 2019

*Correspondence to: Dr. Mayank R. Dhore, Email: mayankdhore@gmail. com

Copyright: (C) the author(s), publisher and licensee Medip Academy. This is an openaccess article distributed under the terms of the Creative Commons AttribUrinary Tract Infectionon Non-Commercial License, which permits unrestricted non-commercial use, distribUrinary Tract Infectionon, and reproduction in any medium, provided the original work is properly cited.

\begin{abstract}
Background: This study was undertaken to investigate and compare the antimicrobial effect of Onion bulbs (Allium cepa) and cinnamon bark (Cinnamomum zeylanicum) against some common bacteria causing Urinary tract infection.

Methods: Antimicrobial activity of the plant extract in different concentrations was observed. Commonly isolated bacteria from the urine samples of suspected and untreated patients which were found to be E. coli, Klebsiella pneumoniae and Pseudomonas aeruginosa.

Results: The comparison between zones of inhibition showed a statistically significant data of both Allium cpea and Cinnamomum zeylanicum. The antimicrobial activity of cinnamon extract showed the maximum effect against Klebsiella pneumoniae, zone of inhibition $25.50 \mathrm{~mm} \pm 3.72$ with $2 \pm 1$ of extract and E. coli with zone of inhibition $11.72 \mathrm{~mm} \pm 1.86$. The onion (Allium cepa) extract exhibited some antimicrobial effect, it was most effective against $E$. coli, though the effect was minimal.

Conclusions: From the current study we conclude that the extract of onion and cinnamon bark are promising but were not as effective as the conventional antibiotics such as ciprofloxacin and nitrofurantoin but can be a good alternative in selected group of patient.
\end{abstract}

Keywords: Allium cepa, antimicrobial activity, Cinnamomum zeylanicum, plant extract, urinary tract infection

\section{INTRODUCTION}

Urinary tract infection (UTI) is a common and painful bacterial disease occurring in all age groups. It affects about 40 to $50 \%$ of women population of reproductive age groups. The increased risk of urinary tract infection in specific subpopulation majorly includes infants, pregnant women, the elderly patients with diabetes, catheterized patients with serious injuries, acquired immunodeficiency syndrome patients and patients with underlying urological abnormalities. In pregnant women the treatment becomes difficult due to restricted use of antimicrobial therapy. urinary tract infection is also common in rural areas of India, this may be attributed to lack of personal hygiene. In such areas the major problem faced by clinicians and the patients is burden of cost of medicine leading to lack of compliance by patients, which further results in drug resistance.

Most common causative organisms observed is Escherichia coli, a commensal found in rectum is responsible for maximum incidences of urinary tract infection. Many studies suggested that $E$. coli was the most common isolated microorganism $37-44 \%$ in cases of 
urinary tract infection, Infrequent causative agents of urinary tract infections include bacteria such as Staphylococcus aureus, Gardnerella vaginalis, Corynebacterium and Lactobacilli, yeasts such as Candida. High level of resistance was shown by these isolates to commonly prescribed antibiotics specially Fluoroquinolones. ${ }^{1}$

There is a considerable variation among general practitioners in the management of patients presenting with symptoms of urinary tract infection. ${ }^{2}$ Mild cases of urinary tract infection are managed conservatively with plenty of water and urine alkalinizers, but sever cases require prompt antimicrobial therapy.

Fluoroquinolones e.g. Norfloxacin, Ciprofloxacin are most commonly prescribed antibiotics in treatment of urinary tract infection cases, though less commonly used antimicrobials are Amoxicillin, Gentamicin, Amikacin, Tetracycline, Nitrofurantoin, Cotrimoxazole (sulfamethoxazole + trimethoprim) and penicillin. But especially in pregnancy drug safety is an important concern and a good percentage of pregnant women is not willing to use conventional antibiotics but has inclination towards the use of plant derived products.

The natural products found commonly in kitchen along with other spices is Cinnamon and Onion is the most common vegetable used around the world. The antimicrobial and anti-inflammatory properties of these common household items was well known to mankind since ancient times. ${ }^{3}$

Cinnamon (Cinnamomum zeylanicum) is one such spice that can potentially help to combat the infectious diseases. It also has antidiabetic, antioxidant and antiulcer activity. The bioactive compound was identified as cinnamaldehyde. Which is responsible for the antimicrobial activity of this spice. ${ }^{4}$

In folk medicine as alternative medication, onion has been used for asthma, bronchitis, whooping cough and similar ailments. Other uses include the treatment of stingray wounds, warts, acne, appetite loss, urinary tract disorders and indigestion.

Onion (Allium cepa) is one of the most commonly found vegetable in the world, it has wound healing properties, used for hair regrowth and antimicrobial effect as well, the active compounds: a flavinol compound Quercetin along with its glycosides and allicin an organosulfer compound, these chemicals of onion are responsible for their antifungal, antibacterial and antiviral properties

The present study is undertaken to determine the antimicrobial activity of Cinnamomum Zeylanicum and Allium Cepa against some common bacteria causing urinary tract infection. These kitchen herbs are with known and presumed antimicrobial activity already in use. And hence this project was undertaken to explore and evaluate their antimicrobial activity to add on the list of easily accessible medicine with almost no side effects as compared to the allopathic counterpart.

\section{METHODS}

\section{Locus}

The present study was undertaken in the Department of Pharmacology, Jawaharlal Nehru Medical College, Sawangi (Meghe), Wardha, Maharashtra.

The Phytochemical extraction and identification procedures were done in Central Research Laboratory, Datta Meghe Institute of medical Sciences, Sawangi (Meghe), Wardha, Maharashtra.

The microbiological procedures were executed in the Department of Microbiology, Jawaharlal Nehru Medical College, Sawangi (Meghe), Wardha, Maharashtra, India,

Duration of study was from January 2017 to September 2018.

\section{Study population}

A total of 301 patient were included in the study who were clinically assessed and suspected cases but untreated patients of urinary tract infection and were admitted in ward or ICU of Acharya Vinoba Bhave Rural Hospital, Sawangi (MEGHE), Wardha, Maharashtra, India, 442001. 301 urine samples were taken in this study.

\section{Inclusion criteria}

- Urine samples of suspected untreated cases of Urinary Tract Infection

- Patients who were not prescribed any antimicrobial therapy.

- $\quad$ Patients admitted in In-patient department

\section{Exclusion criteria}

- $\quad$ Suspected cases of Urinary tract infection who were prescribed antimicrobial therapy.

- Patients receiving treatment from outpatient department.

\section{Statistical Analysis}

Statistical analysis was done by using descriptive and inferential statistics using z-test for difference between two means and software used in the analysis was SPSS22.0 version and $\mathrm{p}<0.05$ are considered as level of significance.

\section{Plant material and extraction procedure}

The Bark of Cinnamomum zeylanicum was purchased from Ayurvedic College, Salod, Wardha, India. The barks 
were washed with water, air dried at ambient temperature, then ground into coarse powder in a grinder. For the preparation of ethanolic extracts, $50 \mathrm{~g}$ of coarsely powdered bark of cinnamon was extracted with $250 \mathrm{ml}$ ethanol in soxhlet apparatus optimized at $70^{\circ} \mathrm{C}$ for $8 \mathrm{~h}$ and $\sim 200 \mathrm{ml}$ of product was recovered then air dried in an open glass plate at ambient room temperature.

Onion bulbs were purchased from local vegetable market in Wardha city. The onion bulbs were cleaned of any dirt from outside, the roots were cut using a knife and the tunic layer was removed by hands, $100 \mathrm{gm}$ of onion was grinded to a smooth paste, the paste was then strained. A pinkish white liquid was obtained, $5 \mathrm{ml}$ of extract was mixed with $5 \mathrm{ml}$ of acetone and filtered using whatman filter paper no. 1. It was transferred in a borosilicate glass bottle and stored in a refrigerator at $4^{\circ} \mathrm{C}$ until further use.

\section{Preparation of agar media}

Himedia Hichrome Muller Hinton agar and disposable sterile petri dishes were purchased from local vendor in Wardha, India. A $40 \%$ of agar solution in distilled water was made. The Agar was autoclaved, allowed to cool and poured in sterile petri dishes, cooled further until solidified.

\section{Preparation of culture for antimicrobial susceptibility test}

The research protocol was approved from Institonal Ethic Committee at Datta Meghe Institute of Medical Sciences. (Deemed to be university), Sawangi (M), Wardha.

The morning urine samples of the suspected \& untreated (without antimicrobial therapy) 301 cases of urinary tract infection patients after thorough checking of history were collected after consent from the patients. The samples were centrifuged and the bottom residue of sample were inoculated on the culture media plate by streak culture method, next day the colonies of Escherichia coli, Klebsiella pneumoniae, Pseudomonas aeruginosa were identified with the help of their color on the Hi chrome agar and isolated (Figure 1).

The isolated colonies were incubated in peptone water for $3 \mathrm{~h}$ then inoculated again by lawn culture method on the culture media for antimicrobial activity of Ciprofloxacin, nitrofurantoin, cinnamon extract, cinnamaldehyde standard, onion extract, quercetin standard was observed. Results were analyzed and compared.
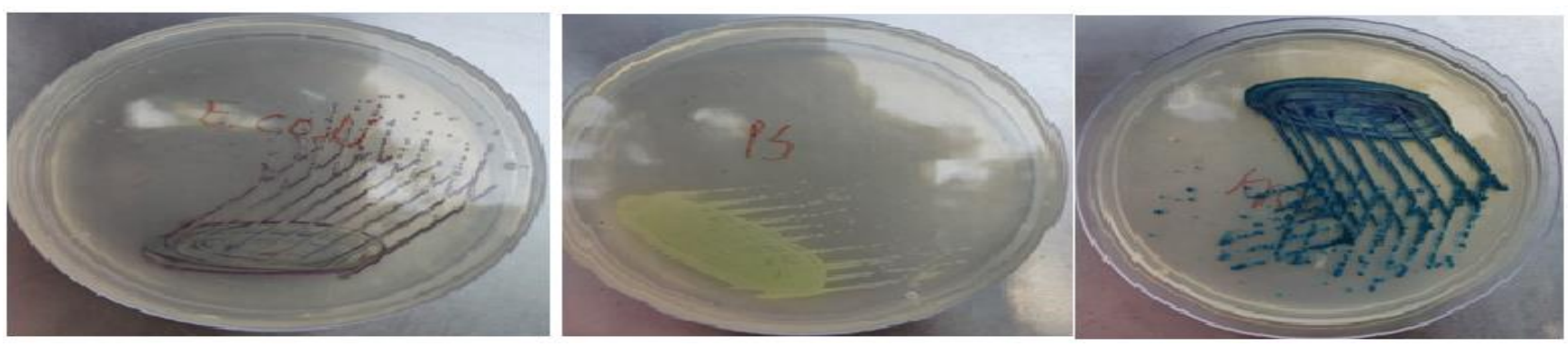

Figure 1: Isolated colonies of $\boldsymbol{E}$. coli. Isolated colonies of Pseudomonas aeruginosa. Isolated colonies of Klebsiella pneumoniae.

\section{Statistical analysis}

was done by using descriptive and inferential statistics using z-test for difference between two means and software used in the analysis was SPSS 22.0 version and $\mathrm{p}<0.05$ is considered as level of significance.

\section{RESULTS}

The mean zone of inhibition (Table 1) of Ciprofloxacin against E.coli was $32.55 \mathrm{~mm} \pm 2.48$, for Nitrofurantoin $21.02 \mathrm{~mm} \pm 2.05$ and Cinnamon extract $11.72 \pm 1.86$, for Cinnamaldehyde $24.39 \mathrm{~mm} \pm 4.05$, for onion extract 6.29 $\mathrm{mm} \pm 1.23$ and for quercetin $14.58 \pm 1.86 \mathrm{By}$ using Z-test for the difference between 2 means, statistically significant difference was found in mean zone of inhibition between ciprofloxacin and nitrofurantoin in comparison with cinnamon extract, cinnamaldehyde, onion extract and quercetin (Figure 2).

The mean zone of inhibition of ciprofloxacin against Klebsiella pneumoniae was $32.03 \mathrm{~mm} \pm 2.38$, for nitrofurantoin $27.01 \mathrm{~mm} \pm 2.34$, for cinnamon extract 25.50 $\mathrm{mm} \pm 3.72$, for cinnamaldehyde $18.45 \pm 3.42$, for onion extract $0.30 \pm 0.73$ and for quercetin $10.22 \pm 1.63$ by using Z-test for difference between 2 means, statistically significant difference was found in mean zone of inhibition (Table 2) between ciprofloxacin and nitrofurantoin in comparison with cinnamon extract, cinnamaldehyde, onion extract and quercetin (Figure 3). 
Table 1: Zone of Inhibition in mm of ciprofloxacin, nitrofurantoin, cinnamon extract, cinnamaldehyde standard $\mathbf{9 8 \%}$, onion extract and quercetin standard $98 \%$ against $E$. coli.

\begin{tabular}{|lllllll|}
\hline & $\mathbf{N}$ & Mean $(\mathbf{m m})$ & Std. deviation & Std. Error Mean & Z-value & p-value \\
\hline Ciprofloxacin & 301 & 32.55 & 2.48 & 0.14 & 116.32 & $0.0001, \mathrm{~S}$ \\
\hline Nitrofurantoin & 301 & 21.02 & 2.05 & 0.11 & 58.24 & $0.0001, \mathrm{~S}$ \\
\hline Cinnamon extract & 301 & 11.72 & 1.86 & 0.10 & - & - \\
\hline Cinnamaldehyde & 301 & 24.39 & 4.05 & 0.23 & - & - \\
\hline Onion Extract & 301 & 6.29 & 1.23 & 0.07 & - & - \\
\hline Quercetin & 301 & 14.58 & 1.86 & 0.10 & - & - \\
\hline
\end{tabular}

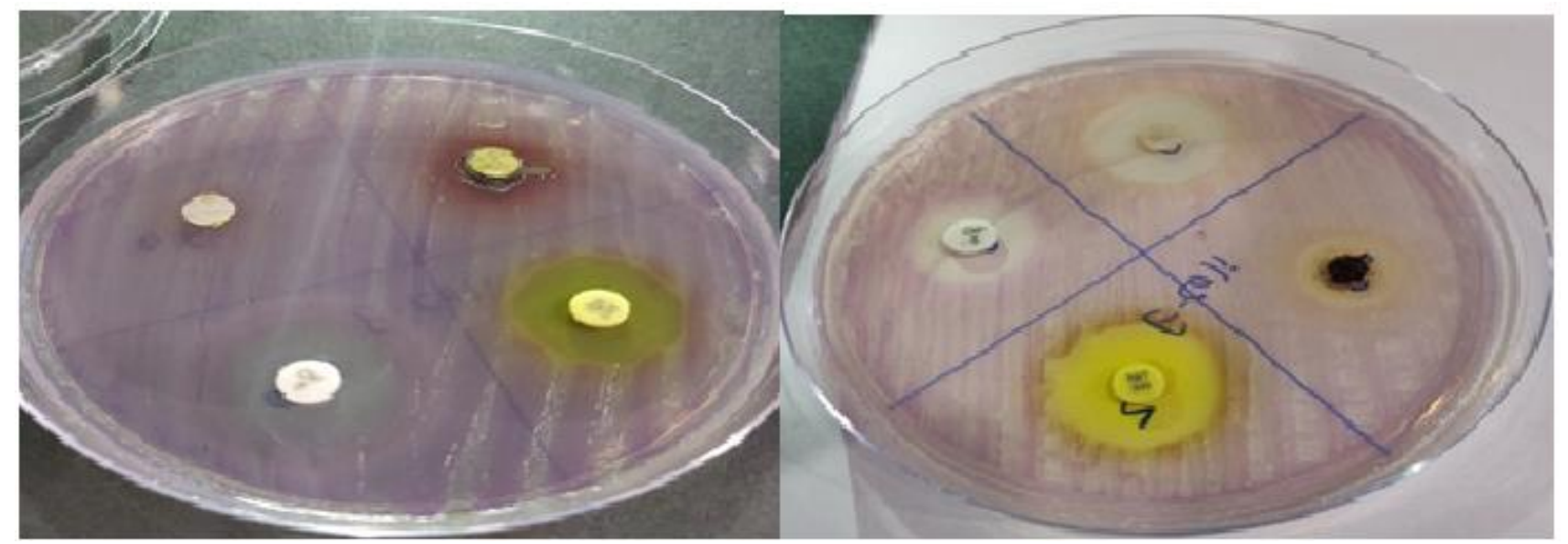

Figure 2: Zones of inhibition of ciprofloxacin, nitrofurantoin, onion extract, quercetin against $E$. coli. Zones of inhibition of ciprofloxacin, nitrofurantoin, cinnamon extract, cinnamaldehyde against $E$. coli.

Table 2: Zone of inhibition in mm of ciprofloxacin, nitrofurantoin, cinnamon extract, cinnamaldehyde standard $\mathbf{9 8 \%}$, onion extract and quercetin standard $98 \%$ against Klebsiella pneumoniae.

\begin{tabular}{|lllllll|}
\hline & N & Mean & Std. Deviation & Std. Error Mean & z-value & p-value \\
\hline Ciprofloxacin & 301 & 32.03 & 2.38 & 0.13 & 25.65 & $0.0001, S$ \\
\hline Nitrofurantoin & 301 & 27.01 & 2.34 & 0.13 & 5.98 & $0.0001, S$ \\
\hline Cinnamon Extract & 301 & 25.50 & 3.72 & 0.21 & - & - \\
\hline Cinnamaldehyde & 301 & 18.45 & 3.42 & 0.19 & - & - \\
\hline Onion Extract & 301 & 0.30 & 0.73 & 0.04 & - & - \\
\hline Quercetin & 301 & 10.22 & 1.63 & 0.09 & - & - \\
\hline
\end{tabular}

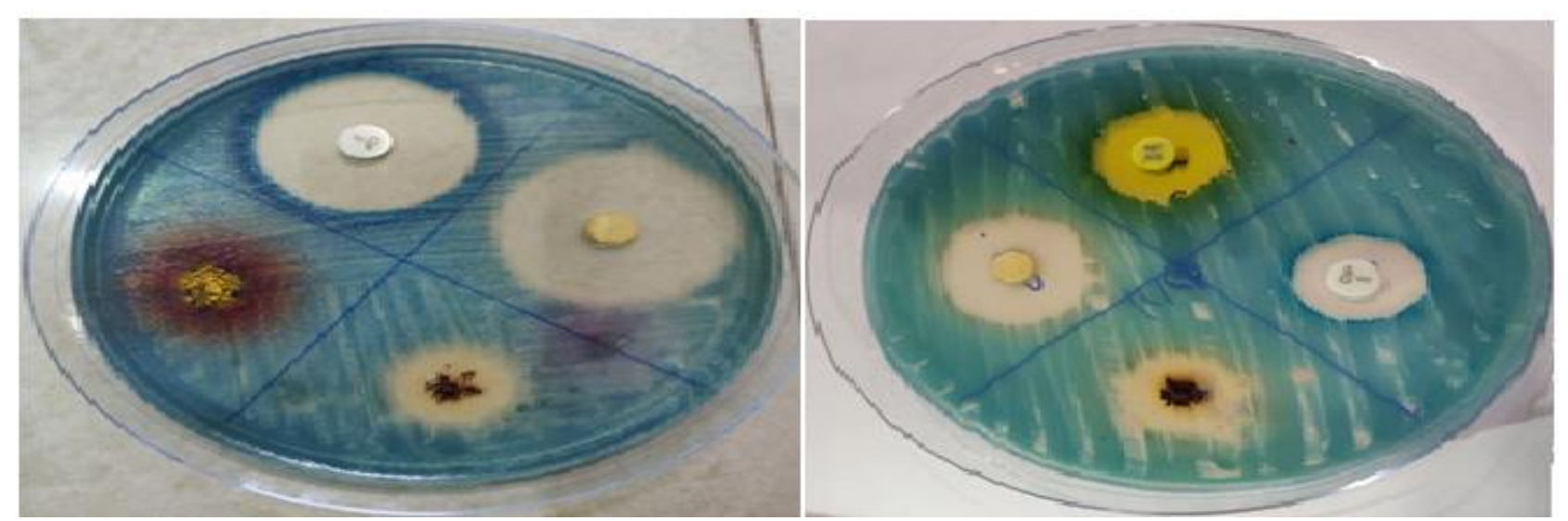

Figure 3: Zone of inhibitions of ciprofloxacin, quercetin, cinnamaldehyde and cinnamon extract against Klebsiella pneumoniae. Zone of inhibitions of ciprofloxacin, nitrofurantoin, cinnamon extract and quercetin against Klebsiella pneumoniae. 
Table 3: Zone of Inhibition in mm of ciprofloxacin, nitrofurantoin, cinnamon extract, cinnamaldehyde standard 98\%, onion extract and quercetin standard $98 \%$ against Pseudomonas aeruginosa.

\begin{tabular}{|lllllll|}
\hline & N & Mean & Std. Deviation & Std. error mean & Z-value & P-value \\
\hline Ciprofloxacin & 301 & 38.50 & 2.16 & 0.12 & 84.06 & $0.0001, \mathrm{~S}$ \\
\hline Nitrofurantoin & 301 & 23.25 & 4.33 & 0.24 & 10.04 & $0.0001, \mathrm{~S}$ \\
\hline Cinnamon Extract & 300 & 20.16 & 3.10 & 0.17 & - & - \\
\hline Cinnamaldehyde & 301 & 14.82 & 3.01 & 0.17 & - & - \\
\hline Onion Extract & 301 & 5.06 & 0.24 & 0.01 & - & - \\
\hline Quercetin & 301 & 14.30 & 1.96 & 0.11 & - & - \\
\hline
\end{tabular}
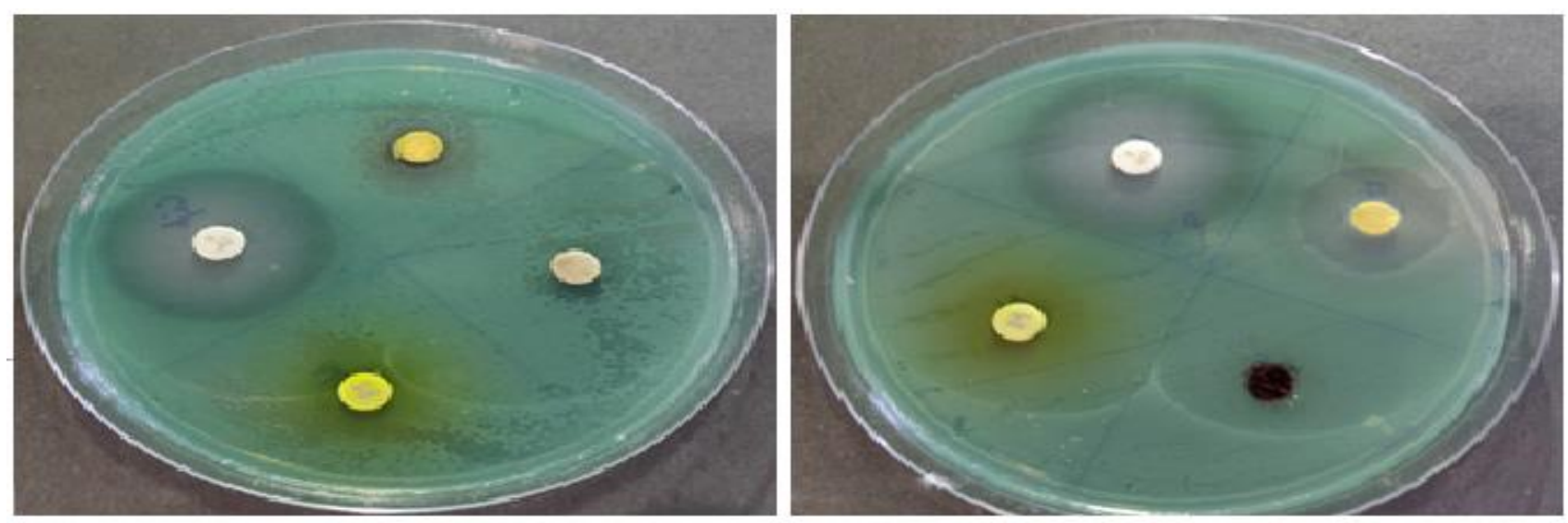

Figure 4: Zones of inhibition of ciprofloxacin, nitrofurantoin, onion extract, quercetin against Pseudomonas aeruginosa. Zones of inhibition of ciprofloxacin, nitrofurantoin, cinnamon extract, cinnamaldehyde against Pseudomonas aeruginosa.

The mean zone of inhibition of ciprofloxacin against Pseudomonas aeruginosa (Table 3) was $38.50 \mathrm{~mm} \pm 2.16$, for nitrofurantoin it is $23.25 \mathrm{~mm} \pm 4.33$ and for cinnamon extract $20.16 \mathrm{~mm} \pm 3.10$, for cinnamaldehyde is 14.82 $\mathrm{mm} \pm 3.01$, for onion extract is $5.06 \mathrm{~mm} \pm 0.24$, for quercetin is $14.30 \pm 1.96$ by using Z-test for difference between 2 means, statistically significant difference was found in mean zone of inhibition between ciprofloxacin and nitrofurantoin in comparison with cinnamon extract, cinnamaldehyde, onion extract and quercetin.(Figure 4).

\section{DISCUSSION}

The present study was undertaken to investigate and compare the antimicrobial effect of Allium cepa and Cinnamomum zeylanicum against some common bacteria causing urinary tract infection. The antimicrobial activity of the cinnamon extract was due to the presence high concentration of cinnamaldehyde which was about $85.06 \% \mathrm{w} / \mathrm{w}$. The susceptibility test disc containing the standard of cinnamaldehyde $98 \%$ was placed over the inoculated culture media, thus confirming that the antimicrobial activity of the cinnamon extract was due to the presence of cinnamaldehyde and the presence of quercetin was thought be the reason for antimicrobial property.
In the current study the mean zone of inhibition of cinnamon extract against $E$. coli was $11.72 \mathrm{~mm} \pm 1.86$. On the contrary to above findings another study conducted by B. Kaskatepe et al, observed that mean zone of inhibition of cinnamon extract against $E$. coli was $38 \mathrm{~mm} .5$

In this study the mean zone of inhibition of cinnamaldehyde against E.coli was $24.39 \mathrm{~mm} \pm 4.05$, while another study conducted by Gupta et al. in 2008 the mean zone of inhibition of cinnamaldehyde against E.coli was found to be $16 \mathrm{~mm} .{ }^{6}$ This variation in the results may be due to different manufacturing processes of the standard.

In this study the mean zone of inhibition of onion extract against $E$. coli against was observed to be $6.29 \mathrm{~mm} \pm 1.23$ however completely contrary to the above finding another study conducted be J. Santas et al, observed no antimicrobial activity of onion extract against $E$. coli. ${ }^{7}$

From It was observed in this study that, the mean zone of inhibition of Quercetin against E.coli against was 14.58 $\mathrm{mm} \pm 1.86$, in the similar study conducted by be J. Santas et al. in 2010 observed the mean zone of inhibition obtained by quercetin was $9.8 \mathrm{~mm} \pm 0.6 .7$ This difference in the observations may be because of the different solvents used by the researchers. 
Current study has observed that the mean zone of inhibition of cinnamon extract against Klebsiellia pneumoniae was $25.50 \mathrm{~mm} \pm 3.72$ similarly, the study conducted by Chang et al. in 2001 also found that cinnamon extract shows inhibitory activity against Klebsiella Pneumoniae through different parameters. ${ }^{8}$

In this study the mean zone of inhibition of cinnamaldehyde against Klebsiellia pneumoniae was $18.45 \mathrm{~mm} \pm 3.42$, in another study conducted by Dhara and Tripathi et al, observed that most study samples of bacterial isolates inhibited between the range of 21-30 mm. ${ }^{9}$ Thus both studies have observed the antibacterial activity of cinnamaldehyde.

In the current study the mean inhibition zone of onion extract against Klebsiella pneumoniae was found to be $0.30 \mathrm{~mm} \pm 0.73$, similarly on study carried out by Lekshmi N. C. J et al. in 2015 observed similar results and the water extract of onion displayed no antimicrobial activity against Klebsiella pneumoniae. ${ }^{10}$ In the above study the mean zone of inhibition of quercetin was found to be $10.22 \mathrm{~mm} \pm 1.63$, the study conducted by Elzbieta Woenicka et al, also found that quercetin shows inhibitory activity against Klebsiella Pneumoniae through different parameters. ${ }^{11}$

In this study the mean zone of inhibition of cinnamon extract against Pseudomonas aeruginosa was found to be $20.16 \mathrm{~mm} \pm 3.10$, similarly in the study carried out by Grullon et al, observed that the inhibition zone of Cinnamon extract against Pseudomonas aeruginosa was 25. mm. ${ }^{12}$ In the present study the zone of inhibition of cinnamaldehyde against Pseudomonas aeruginosa was found $14.82 \mathrm{~mm} \pm 3.01$, on the contrary study done by Kaskatepe et al. in the year 2016 observed that the zone of inhibition of cinnamaldehyde against Pseudomonas aeruginosa was more than $22 \mathrm{~mm}$. This variation in the results may be due to different manufacturing processes of the standard..$^{5,13}$

In this study it was observed that the mean zone of inhibition of onion extract against Pseudomonas aeruginosa was $5.06 \mathrm{~mm} \pm 0.24$, similarly in a study conducted by $\mathrm{N}$ Azu et al, found that the zone of inhibition of onion extract against Pseudomonas aeruginosa was $17 \mathrm{~mm} .{ }^{14}$ In the current study the mean zone of inhibition of quercetin against Pseudomonas aeruginosa was found to be $14.30 \mathrm{~mm} \pm 1.96$, Jaisinghani et al, conducted similar study and found the antimicrobial effect of quercetin against Pseudomonas aeruginosa through different parameters.

\section{CONCLUSION}

We conclude with our study that the phytochemicals have different activity against some common bacteria which cause urinary tract infection. The comparison between zones of inhibition showed a statistically significant data of both Allium cpea and Cinnamomum zeylanicum. The antimicrobial activity of cinnamon extract showed the maximum effect against the bacteria causing urinary tract infection and maximum activity was seen against Klebsiella pneumoniae and E. coli.

The onion (Allium cepa) extract exhibited some antimicrobial effect, it was most effective against $E$. coli, though the effect was minimal. Cinnamon bark (Cinnamomum zeylanicum) extract showed promising antimicrobial activity against Klebsiella pneumoniae but it was also effective against E. coli and Pseudomonas aeruginosa. From the current study we also conclude that the extract of onion and cinnamon bark were not as effective as the conventional antibiotics such as ciprofloxacin and nitrofurantoin but can be a good alternative in selected group of patient. Further extensive preclinical and clinical studies are required to evaluate the safety, efficacy and dose range in human subjects.

Funding: Funding sources from Indian Council of Medical Research (ICMR)

Conflict of interest: None declared

Ethical approval: The study was approved by the Institutional Ethics Committee

\section{REFERENCES}

1. Kaur N, Sharma S, Malhotra S, Madan P, Hans C. Urinary Tract Infection: Aetiology and Antimicrobial Resistance Pattern in Infants From A Tertiary Care Hospital in Northern India. J Clin Diagn Res. 2014 Oct;8(10):DC01-3.

2. Fenwick EA, Briggs AH, Hawke CI. Management of urinary tract infection in general practice: a costeffectiveness analysis. $\mathrm{Br}$ J Gen Pract. 2000 Aug;50(457):635-9.

3. Han X, Parker TL. Antiinflammatory Activity of Cinnamon (Cinnamomum zeylanicum) Bark Essential Oil in a Human Skin Disease Model. Phytother Res. 2017 Jul;31(7):1034-8.

4. Bassolé IHN, Juliani HR, Bassolé IHN, Juliani HR. Essential Oils in Combination and Their Antimicrobial Properties. Molecules. 2012 Apr 2;17(4):3989-4006.

5. Kaskatepe B, Kiymaci ME, Simsek D, Erol HB, Erdem1 SA. Comparison of the Contents and Antimicrobial Activities of Commercial and Natural Cinnamon Oils. Ind J Phar Urina Tra Infect Scien. 2016 Jul 20;78(4):541-6.

6. Gupta C, Garg AP, Uniyal RC, Kumari A. Comparative analysis of the antimicrobial activity of cinnamon oil and cinnamon extract on some foodborne microbes. AJMR. 2008 Sep 30;2(9):247-51.

7. Santas J, Almajano MP, Carbó R. Antimicrobial and antioxidant activity of crude onion (Allium cepa, L.) extracts. Int J Food Science Technol. 2010 Feb 1;45(2):403-9.

8. Chang ST, Chen PF, Chang SC. Antibacterial activity of leaf essential oils and their constituents from Cinnamomum osmophloeum. J Ethnopharmacol. 2001 Sep 1;77(1):123-7. 
9. Dhara L, Tripathi A. Antimicrobial activity of eugenol and cinnamaldehyde against extended spectrum beta lactamase producing enterobacteriaceae by in vitro and molecular docking analysis. Eur J Integra Med. 2013 Dec 1;5(6):527-36.

10. Packia Lekshmi N, Viveka S, Jeeva S, Raja Brindha J. Efficacy of crude extracts of Allium sativum and Allium cepa against human pathogens. Pelagia Research Library Advances in Applied Science Research. 2015:6(1):72-8.

11. Woźnicka E, Kuźniar A, Nowak D, Nykiel E, Kopacz M, Gruszecka J, et al. Comparative study on the antibacterial activity of some flavonoids and their sulfonic derivatives. Acta Pol Pharm. 2013 Jun;70(3):567-71.

12. Djara MJ. Performance measurement of district level hospitals in low income countries: participatory development of an instrument to assess inputs, processes, and outputs for evidence-based management and quality improvement (Doctoral dissertation, Boston University).

13. Kaskatepe B, Kiymaci ME, Suzuk S, Erdem SA, Cesur S, Yildiz S. Antibacterial effects of cinnamon oil against carbapenem resistant nosocomial Acinetobacter baumannii and Pseudomonas aeruginosa isolates. Industrial Crops and Products. 2016 Mar 1;81:191-4.

14. Azu NC, Onyeagba RA. Antimicrobial Properties Of Extracts Of Allium cepa (Onions) And Zingiber officinale (Ginger) On Escherichia coli, Salmonella typhi And Bacillus subtilis. The Internet Journal of Tropical Medicine [Internet]. 2006 Dec 31 [cited 2018 Oct 14];3(2).

15. Jaisinghani RN. Antibacterial properties of quercetin. Microbiology Research [Internet]. 2017 May 2;8(1). Accessed 15 October 2018.

Cite this article as: Dhore MR, Jha AR

Antimicrobial activity of Allium cepa and Cinnamomum zeylanicum against common bacteria causing urinary tract infections: in vitro study. Int $\mathbf{J}$ Basic Clin Pharmacol 2019;8:1185-91. 